\title{
TITLE: CASE REPORT - INFERIOR MESENTERIC VEIN THROMBOSIS AND COVID-19
}

Aureo do Carmo Filho, Bruno da Silva Cunha

\section{AUTHORS AFFILIATION}

Intensive Care Center of the Hospital Universitário Gaffrée e Guinle, Department of General Medicine, Federal University of the State of Rio de Janeiro - Rio de Janeiro - Brazil.

\section{ABSTRACT}

Since its inception in December 2019, Covid-19 has challenged the global scientific community. Some treatments were used in this infection, but doubts still persist regarding the use of medications ${ }^{1}$. More severe cases complicate with endothelial dysfunction, excess thrombin synthesis and decreased fibrinolysis which, associated with hypoxemia, lead to a hypercoagulability state ${ }^{2}$ and some authors indicate the use of anticoagulants for these ${ }^{3}$. For mild cases, doubts remain regarding this indication. We will report the case of a patient with mild symptoms of covid-19, complicated by inferior mesenteric vein thrombosis.

\section{CASE DESCRIPTION}

A 33-year-old obese patient $(\mathrm{BMI}=32.7)$, without other comorbidities, was admitted to our hospital with complaints of severe low back pain, radiated to the hypogastric region, which had started about 8 hours before admission. There was also a report that 11 days before he had a dry cough, fever of $38.2^{\circ} \mathrm{C}$, headache and fatigue, seeking for emergency medical care 4 days later, when he was submitted to nasopharyngeal swab collection for identification SARS-Cov-2, which was positive. On that date, the use of chloroquine diphosphate (450mg every 12 hours for 1 day and 450mg / day for another 4 days), ivermectin (18mg in a single dose), azithromycin (500mg 1x / day, for 5 days) and amoxicillin + potassium clavulanate (875mg / 125mg every 12 hours, for 7 days), were prescribed and used by the patient.

During care at our Service, the patient presented severe hypogastric pain (9/10), without abdominal distension and / or signs of peritonitis. Vital signs were normal and there was no other change in the patient's clinical examination. Blood tests and computed tomography (CT) scans of the chest and abdomen (with venous contrast) were then requested. Blood tests demonstrated changes in liver enzyme levels were observed (TGO=44U/L TGP=148U/L Gamma$\mathrm{GT}=107 \mathrm{U} / \mathrm{L})$, D-dimer $(879 \mathrm{ng} / \mathrm{mL})$, ferritin $(1570 \mathrm{ng} / \mathrm{mL})$ and ultra-sensitive C-reactive protein $(58.2 \mathrm{mg} / \mathrm{dL})$. Chest CT showed infiltration in a peripheral ground-glass pattern, affecting the right and left lower lobes (about $10 \%$ of the lung parenchyma). The abdomen CT scan showed a diffuse reduction of attenuation coefficients by fatty infiltration in the liver, small lymph nodes with a hypodense center measuring up to $1.1 \times 0.7 \mathrm{~cm}$ anterior to the inferior vena cava and thickening of the fat adjacent to the inferior mesenteric vein path, which had an engorged appearance and a luminal density diffusely reduced in relation to the splenic vein, suggestive of venous thrombosis (Figure 1).

The patient was admitted to our Covid Unit, where he was fasted, hydrated and we administered analgesics and antispasmodics intravenously, in addition to enoxaparin 100mg subcutaneously every 12 hours. About 24 hours after admission, there was complete remission of pain, and an oral diet was started for the patient, under careful observation. After 5 days of parenteral anticoagulant treatment, treatment with oral warfarin was started. Two days later, with the INR 
at 2.3, the patient was discharged from hospital with instructions to return to the hospital after 7 days for a new abdominal CT scan.

\section{DISCUSSION}

Mesenteric venous thrombosis (MVT) is a rare condition, estimated at $0.002-0.06 \%$ of hospital admissions and as the cause of acute mesenteric ischemia in only $2.9 \%$ of cases ${ }^{4}$ Unlike mesenteric arterial thrombosis, MVT is associated with prothrombotic states and primary states of hypercoagulability. When there is simultaneous thrombosis of the mesenteric and portal veins, the most common abdominal inflammatory conditions, myeloproliferative neoplasms and local malignancies (such as hepatocellular carcinoma and pancreatic adenocarcinoma) are the main causes ${ }^{5}$

Thrombosis in atypical sites associated with COVID-19 as in our case has also been described. A series of image reviews show cases of acute mesenteric ischemia in association with COVID-196 and the mechanisms suggested are direct damage, hemodynamic impairment associated with COVID-19 and microvascular, arterial and / or venous thrombosis ${ }^{7}$. Our literature review showed a case of thrombosis of probable microvascular etiology, three cases of mesenteric arterial thrombosis and one case of thrombosis with an arterial and venous component ${ }^{8-12 .}$

Patients hospitalized with COVID-19 typically have a prothrombotic state, with lower levels of antithrombin III and increases in fibrin, fibrin degradation products, fibrinogen and dimers with a parallel increase in inflammation markers such as $\mathrm{C}$-reactive protein (CRP) and ferritin ${ }^{13}$ These markers and, in particular, the significantly elevated D-dimer at admission, are associated with greater severity and high mortality, probably reflecting the activation of coagulation by infection and cytokine storm. ${ }^{2,14}$

Contrary to the classic pattern observed in the disseminated intravascular coagulation (DIC) of bacterial sepsis or trauma, the prolongation of aPTT and / or PT is minimal, thrombocytopenia is mild, usually above $100 \times 10^{9} / \mathrm{L}$ and few cases evolve with bleeding being much more frequent reporting of thrombosis. In one study, only $21.6 \%$ of patients with severe COVID-19 met the sepsis-induced-coagulopathy (SIC) criteria for $\mathrm{DIC}^{2}$

There are reports of an increased incidence of COVID-19-associated thromboembolic disease, especially in patients admitted to the intensive care unit (ICU). In a retrospective single-center study, describing 81 patients with COVID-19 who required ICU admission, 25\% developed deep vein thrombosis (DVT). The strongest correlation was with the elevation of the D-dimer and the best cut-off point to predict the occurrence of DVT was $1,500 \mathrm{ng} / \mathrm{mL}$ (with a sensitivity of $85 \%$ and specificity of $89 \%)^{15}$

In a prospective multicenter French cohort of 150 ICU patients, 64 clinically relevant thrombotic complications (16.7\%) occurred, mainly pulmonary embolism (PE). This occurred despite prophylactic anticoagulation, used in $80.7 \%$ of patients and even in patients with therapeutic doses that were administered at 19.3\%. Patients with COVID-19 and acute respiratory distress syndrome (ARDS) had a higher incidence of pulmonary embolism compared to patients without ARDS associated with COVID-19 (11.7 versus $2.1 \%, p<0.008)^{16}$

A Dutch study ${ }^{17}$ with 184 ICU patients reported a cumulative incidence of venous thromboembolism (VTE) of $27 \%$ (95\% confidence interval, $17 \%$ to $32 \%$ ), despite all patients having utilized thromboprophylaxis, though regimens differed between hospitals and doses varied over time. PE again was the most frequent thrombotic complication occurring in 25 patients ( $81 \%$ of cases). Another Italian cohort ${ }^{18}$ also demonstrated a high incidence of 
thromboembolic complications and in this group, thromboprophylaxis was used in $100 \%$ of ICU patients and in $75 \%$ of patients in the ward. About half of the events occurred within the first 24 hours of hospitalization.

A study that used routine ultrasounds reported a $69 \%$ incidence of VTE in those admitted to the ICU and the proportion was significantly higher in patients treated with prophylactic anticoagulation when compared with the therapeutic dose group ( $100 \%$ vs $56 \%$, respectively, $P$ $=0.03)^{19}$ An Italian study found a VTE rate of $22.2 \%$ and its authors strongly suggested that a screening and monitoring venous ultrasound should be performed in all patients ${ }^{20}$

A retrospective analysis of 2,773 patients from a single center in the United States reported similar hospital mortality of $22.5 \%$ of patients who received therapeutic anticoagulation and $22.8 \%$ of patients who did not receive anticoagulation, but in a subset of 395 patients on mechanical ventilation , $29.1 \%$ who received anticoagulation and $62.7 \%$ who did not receive anticoagulation died. Despite this, the study had important limitations such as the lack of details about the patient's characteristics, indications for starting anticoagulants and descriptions of other therapies that patients received that may have influenced mortality. ${ }^{21}$

Another study in Wuhan with 449 patients showed no difference in overall mortality at 28 days between heparin users and non-users (30.3\% vs $29.7 \%, \mathrm{P}=0.910)$, but in the analysis of subgroups there was benefit in patients with the SIC score $\geq 4(40.0 \%$ vs $64.2 \%, P=0.029)$ or Ddimer> 6 times the upper limit of normal $(32.8 \%$ vs $52.4 \%, P=0.017)$ suggesting that some patients may benefit ${ }^{2}$

\section{CONCLUSION}

The data presented and the strong thrombogenic tendency, including thrombosis in atypical sites as in the case presented here, suggest that prophylactic anticoagulation should be widely considered in the context of the COVID-19 infection ${ }^{3}$ However, this may be insufficient in some cases and there may be a benefit in higher doses for groups at higher risk. Thus, some authors have started to recommend intermediate or therapeutic doses based on the d-dimer level, risk factors or DIC scores ${ }^{22-24}$, but there is still not enough data demonstrating the benefits of monitoring coagulation markers or the safety and efficacy of using therapeutic doses of anticoagulants in those with COVID-19 in the absence of other indications. In a recent review the majority of panel members voted for using prophylactic anticoagulation, although $36,8 \%$ of the experts considered an intermediate or therapeutic dose to be reasonable in cases without DIC and $45,9 \%$ in cases with DIC. ${ }^{25}$ The case also reinforces the need to expand the differential diagnosis of abdominal pain in a patient with suspected SARS-CoV-2 infection. 


\section{REFERENCES}

1. Tobaiqy M, Qashqary M, Al-Dahery S, et al. Therapeutic Management of COVID19 Patients: A systematic review. Infect Prev Pract. 2020;(May):100061. doi:10.1016/j.infpip.2020.100061

2. Tang N, Bai H, Chen X, Gong J, Li D, Sun Z. Anticoagulant treatment is associated with decreased mortality in severe coronavirus disease 2019 patients with coagulopathy. J Thromb Haemost. 2020;18(5):1094-1099. doi:10.1111/jth.14817

3. Thachil J, Tang N, Gando S, et al. ISTH interim guidance on recognition and management of coagulopathy in COVID-19. J Thromb Haemost. 2020;18(5):1023-1026. doi:10.1111/jth.14810

4. Harnik IG, Brandt L. Mesenteric venous thrombosis. Vasc Med. 2010;15(5):407418. doi:10.1177/1358863X10379673

5. Sulger E, Dhaliwal HS, Goyal A, Gonzalez L. Mesenteric Venous Thrombosis. In: ; 2020.

6. Bhayana R, Som A, Li MD, et al. Abdominal Imaging Findings in COVID-19: Preliminary Observations. Radiology. Published online May 2020:201908. doi:10.1148/radiol.2020201908

7. Parry $\mathrm{AH}, \mathrm{Wani} \mathrm{AH}$, Yaseen $\mathrm{M}$. Acute mesenteric ischemia in severe coronavirus19 (COVID-19): possible mechanisms and diagnostic pathway. Acad Radiol. Published online 2020. doi:https://doi.org/10.1016/j.acra.2020.05.016

8. Vulliamy $\mathrm{P}$, Jacob S, Davenport RA. Acute aorto-iliac and mesenteric arterial thromboses as presenting features of COVID-19. Br J Haematol. Published online April 2020. doi:10.1111/bjh.16760

9. A Beccara L, Pacioni C, Ponton S, Francavilla S, Cuzzoli A. Arterial Mesenteric Thrombosis as a Complication of SARS-CoV-2 Infection. Eur J case reports Intern Med. 2020;7(5):1690. doi:10.12890/2020_001690

10. de Barry O, Mekki A, Diffre C, Seror M, Hajjam M El, Carlier R-Y. Arterial and venous abdominal thrombosis in a 79-year-old woman with COVID-19 pneumonia. Radiol case reports. Published online April 29, 2020:10.1016/j.radcr.2020.04.055. doi:10.1016/j.radcr.2020.04.055

11. Gartland RM, Velmahos GC. Bowel Necrosis in the Setting of COVID-19. J Gastrointest Surg. Published online 2020. doi:10.1007/s11605-020-04632-4

12. Azouz E, Yang S, Monnier-Cholley L, Arrivé L. Systemic arterial thrombosis and acute mesenteric ischemia in a patient with COVID-19. Intensive Care Med. Published online 2020. doi:10.1007/s00134-020-06079-2

13. Han H, Yang L, Liu R, et al. Prominent changes in blood coagulation of patients with SARS-CoV-2 infection. Clin Chem Lab Med. Published online March 2020. doi:10.1515/cclm-2020-0188

14. Guan W-J, Ni Z-Y, Hu Y, et al. Clinical Characteristics of Coronavirus Disease 2019 in China. N Engl J Med. 2020;382(18):1708-1720. doi:10.1056/NEJMoa2002032

15. Cui S, Chen S, Li X, Liu S, Wang F. Prevalence of venous thromboembolism in patients with severe novel coronavirus pneumonia. J Thromb Haemost. 
Published online April 2020. doi:10.1111/jth.14830

16. Helms J, Tacquard C, Severac F, et al. High risk of thrombosis in patients with severe SARS-CoV-2 infection: a multicenter prospective cohort study. Intensive Care Med. Published online 2020. doi:10.1007/s00134-020-06062-x

17. Klok FA, Kruip MJHA, van der Meer NJM, et al. Incidence of thrombotic complications in critically ill ICU patients with COVID-19. Thromb Res. Published online April 2020. doi:10.1016/j.thromres.2020.04.013

18. Lodigiani C, lapichino G, Carenzo L, et al. Venous and arterial thromboembolic complications in COVID-19 patients admitted to an academic hospital in Milan, Italy. Thromb Res. 2020;191:9-14. doi:10.1016/j.thromres.2020.04.024

19. Llitjos J-F, Leclerc M, Chochois C, et al. High incidence of venous thromboembolic events in anticoagulated severe COVID-19 patients. J Thromb Haemost. Published online April 2020. doi:10.1111/jth.14869

20. Tavazzi G, Civardi L, Caneva L, Mongodi S, Mojoli F. Thrombotic events in SARSCoV-2 patients: an urgent call for ultrasound screening. Intensive Care Med. Published online April 2020:1-3. doi:10.1007/s00134-020-06040-3

21. Paranjpe I, Fuster V, Lala A, et al. Association of Treatment Dose Anticoagulation with In-Hospital Survival Among Hospitalized Patients with COVID-19. J Am Coll Cardiol. Published online 2020. doi:https://doi.org/10.1016/j.jacc.2020.05.001

22. Marietta M, Ageno W, Artoni A, et al. COVID-19 and haemostasis: a position paper from Italian Society on Thrombosis and Haemostasis (SISET). Blood Transfus. 2020;18(3):167-169. doi:10.2450/2020.0083-20

23. Song J-C, Wang G, Zhang W, Zhang Y, Li W-Q, Zhou Z. Chinese expert consensus on diagnosis and treatment of coagulation dysfunction in COVID-19. Mil Med Res. 2020;7(1):19. doi:10.1186/s40779-020-00247-7

24. Kollias A, Kyriakoulis KG, Dimakakos E, Poulakou G, Stergiou GS, Syrigos K. Thromboembolic risk and anticoagulant therapy in COVID-19 patients: emerging evidence and call for action. Br J Haematol. Published online April 2020. doi:10.1111/bjh.16727

25. Bikdeli B, Madhavan M V, Jimenez D, et al. COVID-19 and Thrombotic or Thromboembolic Disease: Implications for Prevention, Antithrombotic Therapy, and Follow-up. J Am Coll Cardiol. Published online April 2020.

doi:10.1016/j.jacc.2020.04.031 
FIGURE 1: A) Thickening of the fat adjacent to the inferior mesenteric vein path, which had an engorged appearance and a luminal density diffusely reduced in relation to the splenic vein, suggestive of venous thrombosis. B) Zoom of the area.
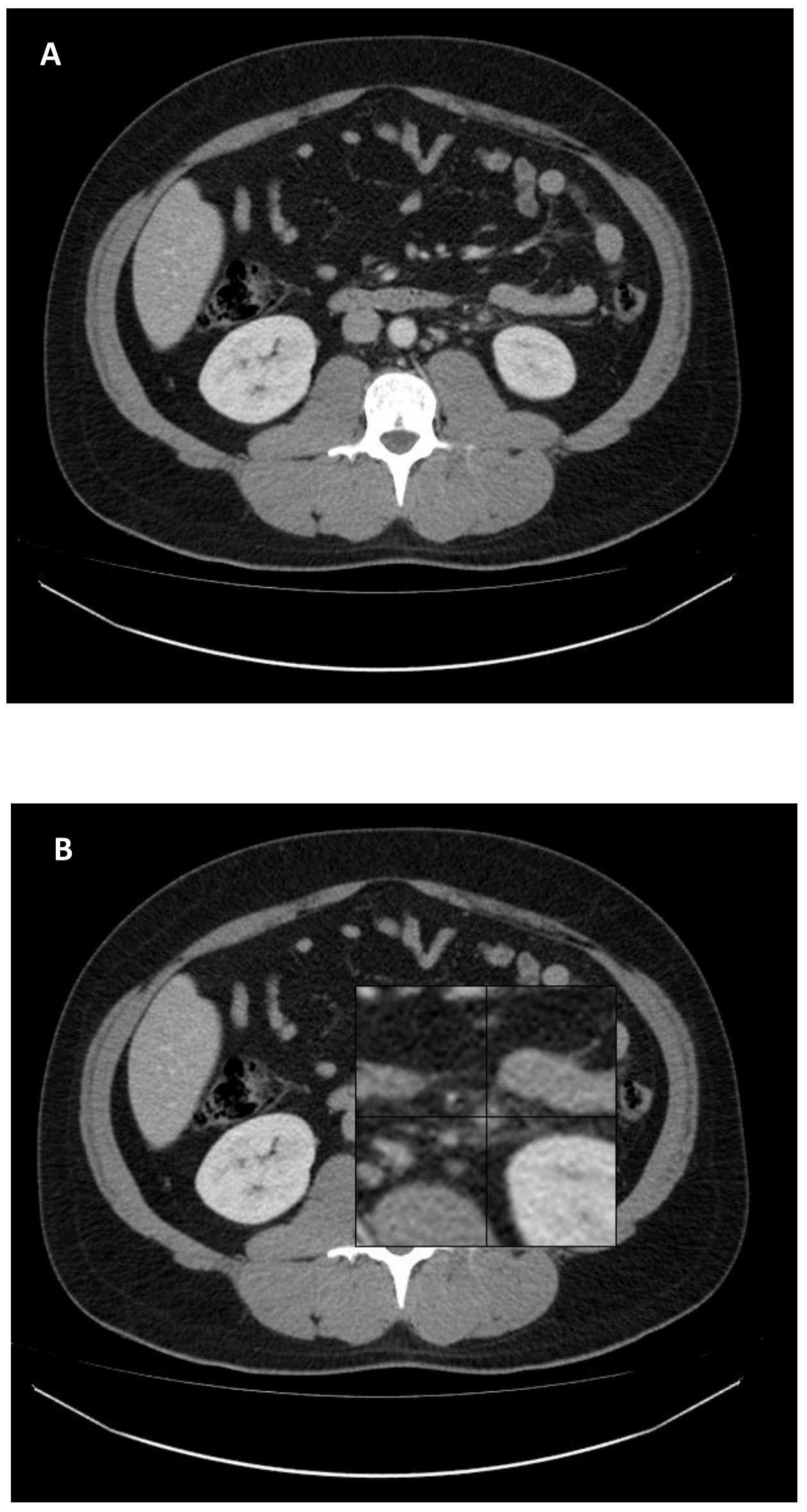